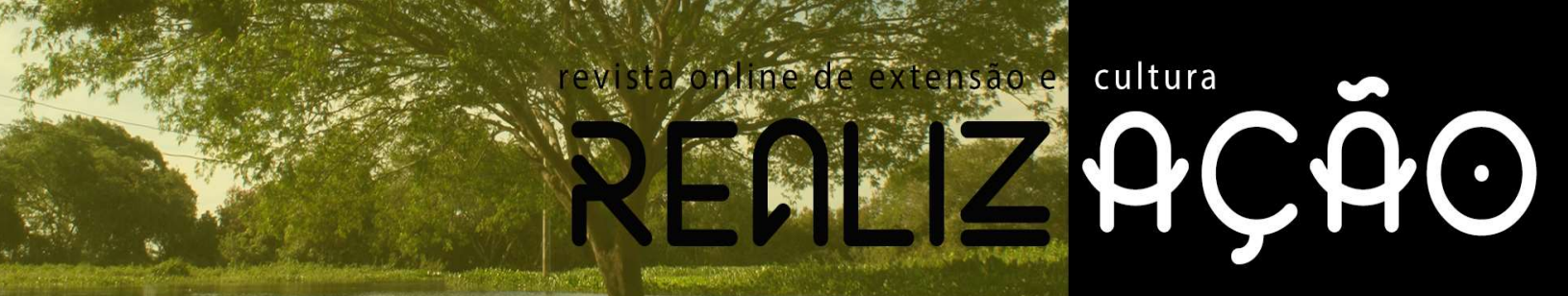

\title{
O papel das Incubadoras Sociais para a promoção da educação popular: uma revisão sistemática de literatura
}

\section{Role of incubators for the promotion of popular education: a systematic literature review}

\author{
Ijean Gomes Riedo
}

DOI

10.30612/re-ufgd.v6i12.926

Recebido em 27/03/2019 Aceito em 07/11/2019

Resumo: As incubadoras tecnológicas de empreendimentos sociais e solidários são espaços de socialização dos conhecimentos produzidos entre o ensino-pesquisa-extensão nas universidades brasileiras. Estas incubadoras tem como princípio a melhoria da qualidade de vida de pessoas em situação de vulnerabilidade social. O objetivo deste artigo é compreender como as incubadoras sociais contribuem para as qualificações transformadoras de grupos sociais - como a educação popular ou a economia solidária. $\mathrm{O}$ estudo teve como metodologia a abordagem de revisão sistemática, descritiva, exploratória e qualitativa, a partir de uma revisão bibliográfica na base de dados Scielo e Emerald. Foram coletados 48 artigos. Destaca-se nos resultados que não houve artigos com interfaces nas duas temáticas (incubadoras sociais e educação popular). Entretanto como discussões, a economia solidária é explicitamente o objeto fundamental de um processo educativo, pautado pela educação popular. As interconexões das pessoas no ambiente das incubadoras sociais vêm desvendando noções práticas de desenvolvimento político-social regional.

Palavras-chave: Incubadora social; Educação popular; Revisão sistemática; Economia solidária.

Abstract: The technological incubators of social and solidarity companies are spaces of socialization of the knowledge produced between teaching-research-extension in the Brazilian universities. These incubators have as principle an improvement in the quality of life of people in situations of social vulnerability. The purpose of this article is how social incubators contribute to the transformative qualifications of social groups - such as popular education or a solidarity economy. The study had as methodology a systematic, descriptive, exploratory and qualitative review approach, based on a bibliographic review in the Scielo and Emerald database. We have collected 48 articles. It stands out in the results that are not articles with interfaces in the two themes (social incubators and popular education). Traditionally, it is a solidarity economy and a fundamental object of an educational process, based on popular education. Communities are not practices of regional political-social development..

Key words: Social incubator; Popular education; Systematic review; Solidarity economy.

\section{Introdução}

A incubação tecnológica de empreendimentos de economia solidária ${ }^{1}$ constitui uma

\footnotetext{
1 São várias as denominações para as Incubadoras Tecnológicas de Empreendimentos de Economia Solidária, como: Incubadora Tecnológica de Cooperativas Populares (ITCP), Incubadora de Tecnológica de Economia Solidária (ITES), Incubadora de Tecnologias Sociais e Solidárias (ITESS), entre outras. Todas tem o mesmo objetivo da melhoria da qualidade de vida para setores sociais excluídos.
} 
das maiores inovações introduzidas no âmbito da extensão universitária brasileira. Centrada na perspectiva do trabalho coletivo e baseada na reflexão sobre educação popular, tecnologia social e qualificação profissional, visando inserir no sistema formal da economia setores marginalizados tanto social quanto economicamente (SCHÜTZ, 2008).

Segundo o relatório final da Secretaria Nacional de Economia Solidária (SENAES) do Ministério do Trabalho e Emprego (MTE), existiam, em 2011, 65 incubadoras tecnológicas de empreendimentos de economia solidária brasileira ${ }^{1}$. No relatório apenas 54 incubadoras foram avaliadas e foram mapeadas por regiões, contando no sudeste com 16 incubadoras, nordeste com 15 , sul com 14, centro oeste com 6 e a região norte com 3 incubadoras. A região sudeste representa o percentual de $29,6 \%$ da quantidade de incubadoras sociais. Os princípios metodológicos centrais dessas incubadoras foram inspirados, principalmente, na proposta de educação popular de Paulo Freire: autogestão, interdisciplinaridade e aprendizado mútuo (IADH, 2011).

Pensadores fazem distinção sobre a educação e a economia, ou seja, a educação se refere aos processos de produção, de reprodução e de trocas de valores de conhecimentos (DURKHEIM, 1952; BOURDIEU e PASSERON, 1982; BRANDÃO, 1995), e a economia se refere a processos de produção, circulação, distribuição e acumulação de bens e serviços materiais (MARX, 1985; MARSHALL, 1987).

Schütz (2008) e Cruz e Guerra (2009) dizem que existe convergência sobre educação popular e economia solidária, ou seja, o ponto em comum em ambas é à necessidade de qualificação. A educação dos setores populares tem prática pedagógica vinculada aos interesses sociais e a economia solidária está orientada nos valores de melhoria de qualidade de vida aos setores sociais excluídos.

Para Gadotti (2001) e Culti, (2006), o processo produtivo está associado ao processo educativo. No entanto, afirmam que o trabalhador só pode estudar trabalhando, fazendo do trabalho, o mediador do saber. Cruz \& Guerra, (2009) atrela as duas variáveis, “os processos educativos são condicionados pelas relações econômicas" - os objetivos da formação educativa está relacionada à reprodução da força de trabalho, à produção de novos conhecimentos ou à qualificação de quadros profissionais e dirigentes - e "os processos econômicos são condicionados pelas práticas educativas" - a qualidade e a capacidade da força de trabalho empregada e/ou do conhecimento e da tecnologia utilizados, a capacidade de inovação e adaptação das estruturas econômicas diante de

1 Não contém na literatura lista ou informações de todas incubadoras para organizar regionalmente no contexto brasileiro. $\mathrm{O}$ estudo apresenta somente as avaliadas no relatório final. 
mudanças e transformações no mercado.

Posto isso, o presente texto problematiza o papel das ITCP's (ou ITES, ou ITESS) na prática da educação popular brasileira. A análise empreendida tem foco na necessidade de compreender como as ações das incubadoras sociais são refletidas nas metodologias e conhecimentos da educação popular, em termos dos seus avanços e limites. Para isso o objetivo deste estudo é analisar a literatura existente sobre essas temáticas, possibilitando sistematizar seus resultados.

\section{Metodologia}

A metodologia utilizada foi à pesquisa aplicada, com abordagens qualitativa, exploratória e descritiva (GIL, 1999). Inicialmente foi realizado busca de dados nas diferentes bases eletrônicas e diante do volume de dados obtidos, optou-se por realizar uma revisão sistemática para a identificação das temáticas relevantes (TRANFIELD et al., 2003).

O processo de revisão sistemática aplicado está ilustrado através de fluxograma (figura 1), o qual descreve as etapas que constituem o processo de elaboração do estudo. Após a definição da pergunta: Como as ações das incubadoras sociais são refletidas nas metodologias e conhecimentos da educação popular, em termos dos seus avanços e limites? Para responder, foi estabelecido o período de investigação da temática entre os anos de 1996-2016. Os artigos foram consultados nas principais bases eletrônicas disponíveis para pesquisa Scielo e Emerald. A pesquisa foi realizada no período de Agosto a Setembro de 2016.

Os critérios estabelecidos para inclusão dos artigos científicos disponíveis foram em formato de $p d f$ e assuntos relacionados aos descritores: educação popular cruzada a incubadora social. Os arquivos selecionados foram salvos no software Mendeley. Para triagem e organização entre os artigos selecionados foi utilizado o Microsoft Excel. Todos os artigos foram separados por aproximação entre os temas abordados pelos autores. 


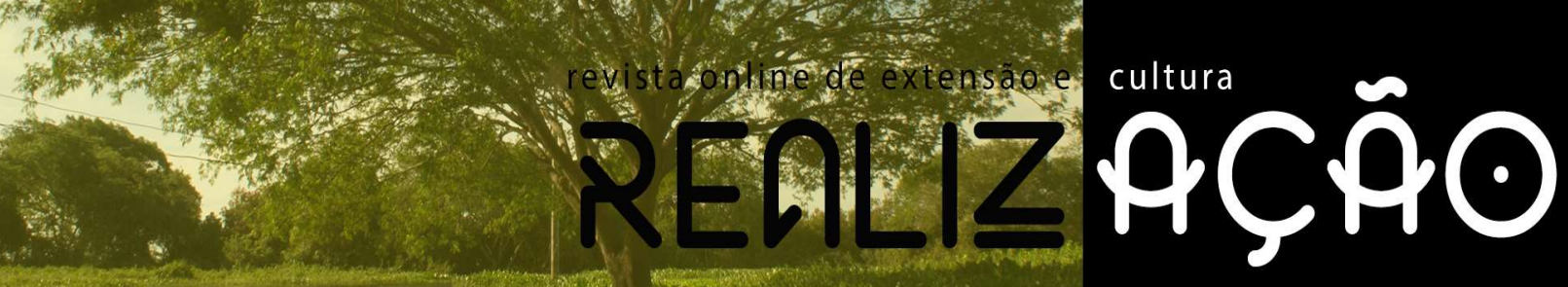

Foram encontrados 282 artigos nas bases pesquisadas, após a leitura dos títulos e resumos dos arquivos, verificaram-se que grande parte dos trabalhos não estavam relacionada a temáticas da pesquisa. Foram encontradas e excluídas 234 pesquisas relacionadas a áreas de ciência da saúde. Após a exclusão, ficaram 48 artigos pertinentes ao foco da Educação Popular e Incubadora Social.

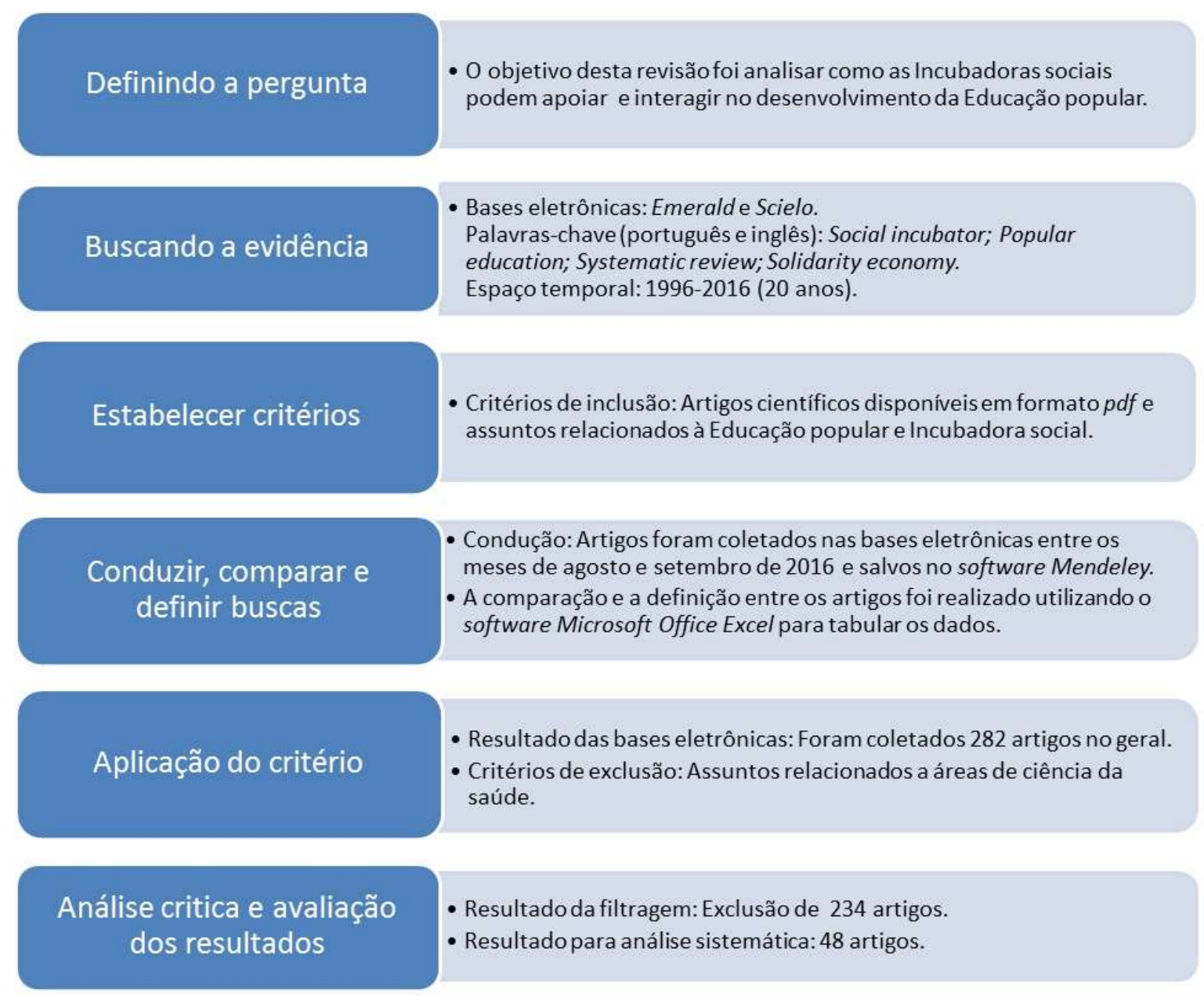

Figura 1 - Descrição do processo de revisão sistemática

Fonte: Elaborado a partir de Tranfield et al. (2003).

\section{Resultados e Discussão}

A economia neoclássica tem teorizado alternativamente como sempre em abundância do empreendedorismo, tornando sua existência não problemática. Através de uma análise das condições que deram origem à organização formal dos coordenadores de projeto de reciclagem universitária nos EUA, isso ampliou a idéia de empreendedorismo coletivo para explicar o 
surgimento de uma organização ocupacional (Lounsbury, 1998).

Durante décadas, desde a primeira classe, em 1945, pela Harvard Business School, os estudiosos se interessaram pelo crescimento explosivo da educação para o empreendedorismo dos negocios empresariais e sociais (figura 2). Os desenvolvimentos economicos e o estado da educação popular para o empreendedorismo e todos descobriram um progresso notável nesse campo (Samwel Mwasalwiba, 2010).

Ainda, tendo demonstrado que os empresários e o empreendedorismo podiam ser encontrados em áreas diferentes dos negócios, implicando que suas conquistas são de importância vital para a economia e a sociedade em geral, algumas reflexões sobre os desafios enfrentados na tentativa de desenvolver mais empresários, e, ao mesmo tempo, promoviam mais empreendedorismo de organizações em todos os lugares do mundo (Thompson, 1999).

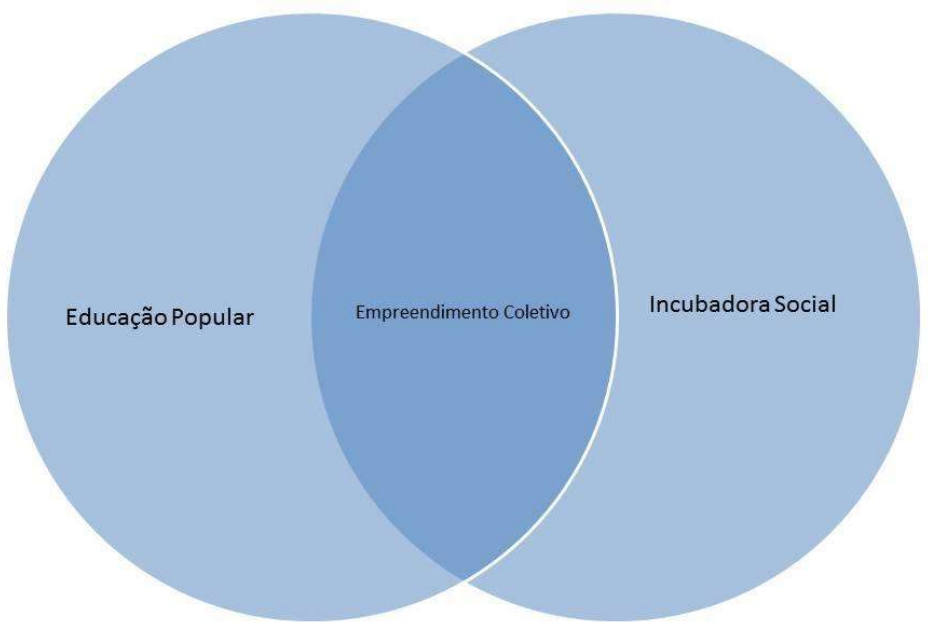

Figura 2 - Análise sistemática do elo entre Educação Popular e Incubadora Social

Fonte: Resultados da pesquisa

O conceito de empreendedorismo social vem emergindo rapidamente nos setores privado, público e sem fins lucrativos nos últimos anos e o interesse pelo empreendedorismo social continua a crescer. O empreendedorismo social tornou-se um fenômeno global que afeta a sociedade, empregando abordagens inovadoras para resolver problemas sociais. Existe um interesse considerável no empreendedorismo social. No entanto, o empreendedorismo social significa coisas diferentes para pessoas diferentes, criando grande confusão na literatura e na prática (Jiao, 2011; Lili, 2011). 
Apesar de ser reconhecido como uma das principais histórias de sucesso no Ensino Superior, as escolas de negócios empresariais e sociais estão em uma encruzilhada em seu desenvolvimento. Atualmente, eles enfrentam uma crise de imagem e identidade e foram sujeitos a uma ampla gama de críticas sobre seu status social como escolas acadêmicas e profissionais (Roper \& Cheney, 2005; Thomas, 2007).

Os profissionais egressos de instituições de ensino superior (IES) entram em um ambiente que está em mudança e instabilidade. A tecnologia e os fatores contingenciam e mudam diariamente o mundo da oportunidade de trabalho (Jarman \& Singh Chopra, 2008; Lili, 2011). Os novos empregos, em sua maioria, são criados em pequenas e médias empresas que não encontram espaços para se desenvolverem, e as grandes corporações geralmente empregam menos pessoas. Isso significa para os graduados do século XXI, que estão sendo desafiados ao constante aprimoramento, onde a noção de emprego seguro não é mais sustentável e onde a noção de que uma educação universitária é o passaporte para o emprego de alto nível (Collins, Hannon, \& Smith, 2004; Williams \& Windebank, 2006).

A necessidade de emprego de carteira assinada torna-se mandatória, imposto pela sociedade capitalista, em face às expectativas dos empregadores estão mudando e a demanda por graduados que apresentem comportamentos e atitudes empresariais está aumentando (Bàculo, 2006; Collins et al., 2004). Uma escala valida para avaliar a orientação de negócios empresariais ou sociais de um indivíduo seria bastante valiosa para professores no ensino de empreendedorismo, bem como na estruturação de métodos de ensino, na tomada de decisões de seleção de equipe e em avaliações de projetos (Langkamp Bolton \& Lane, 2012).

A ampla visão do sucesso que esses jovens profissionais, homens e mulheres, que incluíram trabalho, serviço familiar e comunitário, bem como interesses espirituais e de lazer. No entanto, os indicadores sutis para identidades profissionais de gênero estão lá e serão importantes para acompanhar o futuro. Além disso, essas diferenças de gênero nas atitudes em relação à educação popular e empresarial podem ser facetas de desenvolvimento efetivo no comportamento organizacional, como importante construção de programas economicos e geração de empregos (Iakovleva, Solesvik, \& Trifilova, 2013; Jones, Jones, Packham, \& Miller, 2008).

Entre essas partes interessadas dentro do ambiente organizacional, existe a crença comum de que a educação popular é a chave para o empreendedorismo social e que ajudaria a influenciar a cultura e a construir economias empreendedoras. Sabe-se que, embora não haja consenso nas questões básicas de definição, existe uma compreensão comum do que a educação 
popular para o empreendedorismo social geralmente está tentando alcançar (Jiao, 2011; Roper \& Cheney, 2005; Samwel Mwasalwiba, 2010).

A interação formal e informal entre as profissionais emergiu como uma característica essencial do processo de desenvolvimento de graduados empreendedores nas IES do Reino Unido (Matlay, 2011).

Notadamente a educação popular e o futuro das bibliotecas fisicas estão fortemente ameaçados pelo ambiente em rede, que está transformando as bibliotecas, o ensino superior e o resto da sociedade para as tecnologias de informação (Jackson, Gharavi, \& Klobas, 2006; Li, 2006; Wilson, 2004).

Então, entende-se que o raciocínio para a incubação reside em dois fatos. Primeiro, as pequenas empresas são um componente vital do crescimento econômico e do desenvolvimento social. Em segundo lugar, a taxa de falha das pequenas empresas é alta, especialmente durante os anos formativos. A luz dessas altas taxas de falha de pequenas empresas, por um lado, e as contribuições vitais que elas contribuem para a economia e a sociedade, por outro, numerosos conceitos e estratégias foram formulados para atender essas empresas (Jiao, 2011; Xu, 2009).

\subsection{Cases nos países}

O entendimento da orientação empresarial no nível individual também pode ser valioso para futuros empresários, incubadoras de empresas e sociais e potenciais investidores que estejam considerando apoiar propostas de negócios rurais ou urbanos. No entanto, os traços de personalidade e atitudes contribuem para a probabilidade de uma pessoa estar em um negócio social, ou em sentido inverso, a exposição a atitudes empreendedoras de impacto comercial (Cross, 2000; Langkamp Bolton \& Lane, 2012; Newbery \& Bosworth, 2010; Roper \& Cheney, 2005).

Se uma grande cultura comercial oculta de empresas e empresários que operam total ou parcialmente em consonancia com a realidade em comunidades rurais ou em tipos particulares de comunidades rurais, então pode ser que essas comunidades legitimem a cultura da empresa oculta, poderia ser um meio importante de promover o desenvolvimento empresarial e econômico nessas comunidades (Williams, 2011).

Embora o empreendedorismo tenha sido historicamente teorizado de forma subjulgada ou socializada, o conceito de empreendedorismo coletivo desloca a atenção para uma visão mais integrada da ação que se concentra em como os processos sociais do nível do solo em contextos históricos particulares restringem e permitem o surgimento da atividade empreendedora (Jiao, 2011; Lounsbury, 1998). 
O empreendedorismo tornou-se sinônimo de êxito e competitividade locais, regionais e nacionais. O tema dominante baseia-se na hipótese de que uma maior e melhor educação para o empreendedorismo afetará positivamente o desenvolvimento de atitudes empresariais, habilidades, habilidades e intenções dos estudantes para criar novas empresas, o que, por sua vez, resultará em um crescimento comparável da atividade empresarial (Piperopoulos, 2012).

Na América do Sul, a Argentina está agrupada com economias orientadas para a eficiência social e ocupa o terceiro lugar na atividade em fase inicial, atrás do Peru e da República Dominicana, mas acima do México e do Brasil (Helms, Rodríguez, Ríos, \& Hargrave, 2011). É expressivamente visto que a opinião popular é refletida sobre as recentes mudanças na demanda de tecnologia nos principais países industrializados determinam a transformação da economia "regulamentada" desde as décadas de 1950 e 1960, dominada por empresas de gestão para a Economia "empreendedora" dos anos 80 e 90, dominada por pequenas empresas (Iacobucci \& Micozzi, 2012).

Na China a primeira incubadora criada foi o Centro de Pioneiros de Wuhan Donghu, em junho de 1987, seguindo a linha de um especialista visitante da ONU que fez uma sugestão à Comissão de Ciência e Tecnologia do Estado. Desde então, os programas de incubação de empresas têm sido utilizados como um dos principais instrumentos de política do Programa Torch, um programa nacional destinado a criar um ambiente apropriado para o desenvolvimento de indústrias de alta tecnologia na China (Xu, 2009).

Ainda o governo chinês através das empresas estatais coletivas, iniciou estruturação das empresas denominadas como município-aldeia, que distinguem de empresas privadas genuínas, onde pudessem desenvolver através de educação popular e a inserção social de sua população no mercado. A economia chinesa passou por um processo de transformação de uma economia centralizada para uma economia de mercado socialista (Modderman, Gorter, Dalhuisen, \& Nijkamp, 2007).

Já na Palestina, embora existam várias tensões decorrentes da implantação de políticas de iniciativa de desenvolvimento das comunidades, inclui-se um desafio distinto a uma noção tradicional de educação universitária, somam-se justificativas suficientes para a base do desenvolvimento e a extensão no seu modelo de organização estatal. Existe um debate crescente sobre o papel que as universidades devem desempenhar em suas comunidades, seja como educadores ou formadores de seus alunos. Aqueles que defendem que as universidades sejam instalações de treinamento para graduados em tecnologia argumentam que as universidades não 
estão respondendo rapidamente o suficiente para as realidades do mercado em negócios e tecnologia que estão ocorrendo em uma taxa acelerada e que redefinem o papel dos sistemas de informação nos negócios e organizações atuais (Rabayah \& Sartawi, 2008).

A manutenção do capital humano não só indica que a empresa alcançou um nível adequado de produtividade, mas também sugere uma melhora em várias outras medidas, como habilidades dos funcionários, criatividade, reinvenção e renovação institucional, empreendedorismo, nível de capacitação, compromisso e satisfação no trabalho (Drew, 2011; Sagie \& Weisberg, 2001). Assim, dentro de um ambiente em rápida mudança, o desafio de muitos gerentes israelenses de recursos humanos foi projetar e introduzir valores e estratégias para enriquecer a organização (Sagie \& Weisberg, 2001).

Nesse aspecto, o governo do Reino Unido tem incentivado o empreendedorismo criando ambiente em que essas atividades podem florescer de acordo com as visões globais impostas pelo mundo globalista (Collins et al., 2004). Nas indústrias criativas da Nova Zelândia, como a startup social de designer de moda, transformam essa atividade individual em uma empresa criativa, que diversifique seu foco para que eles possam explorar sua criatividade para obter ganhos comerciais. Em outras palavras, eles devem combinar a criatividade com a inovação e se envolver em empreendedorismo cultural (Mills, 2012).

$\mathrm{Na}$ avaliação polonesa da educação empresarial, o seu impacto e eficácia social é percebida através de atividade empreendedora anteriormente incipiente dentro de um país emergente como da União Européia (UE). Ou seja, existe um debate contínuo na academia sobre a eficácia da educação popular e social formada na empresa e a necessária evidência para validar seu desempenho (Jiao, 2011; Jones et al., 2008).

Como a inovação é inevitavelmente um processo social, esse acoplamento exige que o empreendedor da moda nascente adquira o capital social apropriado para apoiar de forma construtiva seu processo de desenvolvimento empresarial (Mills, 2012; Sullivan, 2011).

A educação para o empreendedorismo é fundamental para o crescimento econômico nos países desenvolvidos e em desenvolvimento em todo o mundo. A totalidade da experiência adquirida pelos alunos nas graduações é e será influenciada por muitos fatores, incluindo as experiências anteriores que tiveram na educação, suas aspirações pessoais para o futuro, suas expectativas em relação à sua vida na universidade e como sua experiência na universidade suporta suas aspirações futuras. Alguns estudantes chegarão à universidade com experiência em negócios e empreendimentos, ou seja, de famílias que gerem negócios. Eles trarão um conjunto único de 
experiências anteriores para seu ambiente educacional (Collins et al., 2004; Peltier \& Scovotti, 2010).

O gerenciamento do setor público na Nova Zelândia foi considerado a partir de várias perspectivas, com uma divisão comum sendo o papel do governo como um remo ou uma direção. Em consonâncias com o papel do governo, os pesquisadores e os empreendedores sociais buscam a promoção do desenvolvimento econômico baseados em uma sociedade estável, popular e progressiva (Luke, 2008; Luke, Kearins, \& Verreynne, 2011).

Para incentivar essa atividade no Reino Unido, tem havido uma série de desenvolvimentos nas universidades, que incluem o desenvolvimento do ensino do empreendedorismo, movimentos específicos para a consultoria, o desenvolvimento de parques científicos, incubadoras de empresas e diversos programas de suporte e spin-out de empresas universitárias (Emslie \& Bent, 2007; Jones et al., 2008; Zhou, 2008).

O empreendedorismo das mulheres na Russia e na Ucrânia foi um fenômeno no impacto de emprego e no ambiente empresarial e social global e, como resultado, a escala e o crescimento do empreendedorismo feminino atraíram muita atenção considerável de acadêmicos, profissionais e formuladores de políticas no mundo (Iakovleva et al., 2013).

A evidência esmagadora da Grã-Bretanha é que o número de pequenas empresas está aumentando substancialmente. No entanto, a maioria das pequenas empresas parece criar empregos apenas para seus proprietários e, em muitos casos, esses trabalhos não são de natureza suficientemente substancial para sustentar o tipo de estilo de vida a que muitos aspirariam. Grande parte da política governamental atual está simplesmente transformando os desempregados em limpadores de janelas autônomas em nome da cultura empresarial (Stanworth \& Stanworth, 1990).

Uma economia capitalista é, de fato, mais rápida para aproveitar oportunidades súbitas e, portanto, tem maior produtividade com inovações por empreendedores apoiados por banqueiros e instituições credíveis. As estruturas capitalistas da África Ocidental parecem diferentes, mas ainda estão profundamente inseridas na economia social e conduzidas por trabalhadores informais que empreendem outros, emprestam da definição original (Minard, 2009).

Com especial referência para examinar as reformas mais recentes da educação e a mudança da governança do ensino superior em Singapura, este artigo enfatiza a forma como o governo de Singapura mudou seus modelos de governança de educação superior para melhorar a competitividade global do seu sistema de ensino superior, adotando mais política pró-concorrência instrumentos e permitindo o crescimento da educação transnacional no estado da cidade (Ho Mok, 
2008).

O ritmo cada vez maior de mudanças que afetam as esferas sociais, política, econômica e organizacional significa que nosso conhecimento, compreensão e habilidades exigem um aumento constante e reestruturação. Os gerentes nas esferas públicas e privada são particularmente afetados, pois enfrentam mudanças incessantes nas práticas e ideias/teorias que afetam seu trabalho (Quinn \& Wennes, 2008).

Em geral, mais e mais universidades estão envolvidas na aprendizagem e na mudança para a sustentabilidade no ensino superior e a melhoria da qualidade tornou-se o foco do trabalho atual (Cao, Zhao, \& Chen, 2009; Niu, Jiang, \& Li, 2010). O estado atual das atividades em educação para o desenvolvimento sustentável na China pode-se inferir que é necessário mais trabalho para atingir os objetivos da educação para o desenvolvimento sustentável, não só no ensino superior, mas também no papel que as universidades adotam a educação para o desenvolvimento sustentável em toda a sociedade (Niu et al., 2010).

O pensamento contemporâneo e a educação popular estão atrelados sobre o futuro dos países e regiões "em desenvolvimento". Muitas vezes profundamente empobrecidos, esses países enfrentam certos conflitos sociais e degradação ambiental, a menos que sejam capazes de se modernizar de forma a conciliar eficiência econômica, equidade social e sustentabilidade ambiental (Macleod, Mcfarlane, \& Davis, 1997).

Embora não haja consenso sobre o conteúdo e a estrutura da educação para o empreendedorismo, os resultados do estudo atual dizem que as universidades devem, pelo menos, encorajar o desenvolvimento de ideias criativas para serem empreendedoras, fornecer o conhecimento necessário sobre empreendedorismo e desenvolver as habilidades empresariais (Shurville, Browne, Whitaker, Shurville, \& Browne, 2009; Turker \& Sonmez Selcuk, 2009).

Da literatura, conhecemos os atributos que os alunos e seus pais procuram ao considerar as universidades sua profissionalização popular, mas com a diminuição dos recursos empreendidos impedem os alunos em sua busca educacional. A literatura também discute os efeitos do crescente consumismo que estão sendo expressos por muitas dos pesquisadores das instituições de ensino superior (Freeman \& Thomas, 2005; Shenkin \& Coulson, 2007).

\section{Considerações}

Muitos estudiosos compartilham a mesma opinião que há um progresso notável feito e, como campo de estudo, alcançou um lugar no mundo de acadêmicos. Este nível de progresso é 
atribuído ao crescente apoio recebido de muitas partes interessadas, incluindo formuladores de políticas, acadêmicos e estudantes. A universidade pode ser uma fonte de processo tecnológico para a economia social pelo menos em casos modestos.

Novamente, há uma diversidade de tipos de educação programas que podem ser agrupados em três em relação ao seu foco e objetivos, ou seja, educar para, sobre ou em empreendedorismo, mas ainda existe uma variação substancial nos métodos de ensino. $\mathrm{O}$ empreendedorismo é ensinado a vários grupos-alvo, desde estudantes até desempregados e grupos minoritários na comunidade. Pode-se afirmar que o empreendedorismo social pode ser promovido como resultado de um processo de aprendizagem.

O papel da educação é crucial no ambiente em rápida evolução de hoje. Dentro desse papel, as universidades são responsáveis por muitos, incluindo os alunos que são educados neste meio, bem como a população onde esses estudantes vivem. Os problemas associados a uma posição liberal em matéria de prestação de contas são tanto políticos como sistêmicos; eles se relacionam não apenas com o clima político dominante, mas também com a natureza problemática da própria comunicação. O último desses problemas tem sido comumente abordado por sociólogos e teóricos da cultura.

\section{Referências Bibliográficas}

Bàculo, L. (2006). Tackling informal employment: the case of southern Italy. International Journal of Manpower, 27(6), 552-571. https://doi.org/10.1108/01437720610690482

Bourdieu, P; Passeron, J. C. A reprodução. Elementos para uma teoria do sistema de ensino. Rio de Janeiro: Francisco Alves, 1975.

Brandão, Carlos (1995). O que é educação?. São Paulo: Brasiliense.

Cao, Y., Zhao, L., \& Chen, R. (2009). Institutional structure and incentives of technology transfer: Some new evidence from Chinese universities. Journal of Technology Management in China, 4(1), 67-84. https://doi.org/10.1108/17468770910942843

Collins, L., Hannon, P. D., \& Smith, A. (2004). Enacting entrepreneurial intent: the gaps between student needs and higher education capability. Education + Training, 46(8/9), 454-463. https://doi.org/10.1108/00400910410569579

Cross, J. (2000). Street vendors, and postmodernity: conflict and compromise in the global economy. International Journal of Sociology and Social Policy, 20(1/2), 29-51. https://doi.org/10.1108/MBE-09-2016-0047 
Cruz, Antonio; GUERRA, Janaína da Silva. In: HERBERT, Sérgio et al. Participação e práticas educativas - a construção coletiva do conhecimento. São Leopoldo: Oikós, 2009. pp. 90-105.

Culti, M. N. (2006). O desafio do processo educativo na prática de incubação de empreendimentos econômicos solidários. Retrieved from http://www.bdae.org.br/dspace/handle/123456789/349

Drew, S. a. W. (2011). Strategic roles of centers in business school development. Journal of Management Development, 30(9), 793-815. https://doi.org/10.1108/02621711111164303

Durkheim, É.. Definição de Educação. In: Educação e Sociologia. 3 ed. Trad Lourenço Filho. São Paulo: Melhoramentos, 1952.

Emslie, L., \& Bent, R. (2007). Public sector business support providers: marketing business support to the ethnic business sector. Marketing Intelligence \& Planning, 25(5), 460-482. https://doi.org/10.1108/02634500710774941

Freeman, I., \& Thomas, M. (2005). Consumerism in education: A comparison between Canada and the United Kingdom. International Journal of Educational Management, 19(2), 153-177. https://doi.org/10.1108/09513540510582444

Guerra, A. C. J. da S. (2009). Educação popular e economia solidária nas incubadoras universitárias de cooperativas populares - práticas dialógicas mediadas pelo trabalho, 90-105.

Helms, M. M., Rodríguez, M. a., Ríos, L. D. L., \& Hargrave, W. (Bill). (2011). Entrepreneurial potential in Argentina: a SWOT analysis. Competitiveness Review: An International Business Journal Incorporating Journal of Global Competitiveness, 21(3), 269-287. https://doi.org/10.1108/10595421111134859

Ho Mok, K. (2008). Singapore's global education hub ambitions. International Journal of Educational Management, 22(6), 527-546. https://doi.org/10.1108/09513540810895444

Iacobucci, D., \& Micozzi, A. (2012). Entrepreneurship education in Italian universities: trend, situation and opportunities. Education + Training, 54(8/9), 673-696. https://doi.org/10.1108/00400911211274828

Iakovleva, T., Solesvik, M., \& Trifilova, A. (2013). Financial availability and government support for women entrepreneurs in transitional economies. Journal of Small Business and Enterprise Development, 20(2), 314-340. https://doi.org/10.1108/14626001311326752

IADH. (2011). Avaliação Do Programa Nacional de Incubadoras Tecnológicas de Cooperativas e Empreendimentos Solidários - PRONINC. Recife: Secretaria Nacional de Economia. http://acesso.mte.gov.br/data/files/8A7C812D3C3A6BBF013CB4C1940C0E82/Avalia

\%C3\%A7\%C3\%A3o\%20do\%20PRONIN\%202011\%20_\%20IADH.pdf. Consultado em no dia 08 de setembro de 2017.

Jackson, P., Gharavi, H., \& Klobas, J. (2006). Technologies of the self: Virtual work and the inner panopticon. Information Technology and People, 19(3), 219-243. https://doi.org/10.1108/09593840610689831 
Jarman, J., \& Singh Chopra, P. (2008). Business services and the knowledge economy in Malaysia. International Journal of Sociology and Social Policy, 28(5/6), 193-203. https://doi.org/10.1108/01443330810881240

Jiao, H. (2011). A conceptual model for social entrepreneurship directed toward social impact on society. Social Enterprise Journal, 7(2), 130-149. https://doi.org/10.1108/17508611111156600

Jones, P., Jones, A., Packham, G., \& Miller, C. (2008). Student attitudes towards enterprise education in Poland: a positive impact. Education + Training, 50(7), 597-614. https://doi.org/10.1108/00400910810909054

Langkamp Bolton, D., \& Lane, M. D. (2012). Individual entrepreneurial orientation: development of a measurement instrument. Education + Training, 54(2/3), 219-233. https://doi.org/10.1108/00400911211210314

Li, X. (2006). Library as incubating space for innovations: practices, trends and skill sets. Library Management, 27(6/7), 370-378. https://doi.org/10.1108/01435120610702369

Lili, Z. (2011). Comparative study of China and USA's colleges entrepreneurship education from an international perspective. Journal of Chinese Entrepreneurship, 3(3), 185-194. https://doi.org/10.1108/17561391111166966

Lounsbury, M. (1998). Collective entrepreneurship: the mobilization of college and university recycling coordinators. Journal of Organizational Change Management, 11(1), 50-69. https://doi.org/10.1108/09534819810369572

Luke, B. (2008). Financial returns from new public management: a New Zealand perspective. Pacific Accounting Review, 20(1), 29-48. https://doi.org/10.1108/01140580810872834

Luke, B., Kearins, K., \& Verreynne, M. (2011). The risks and returns of new public management: political business. International Journal of Public Sector Management, 24(4), 325-355. https://doi.org/10.1108/09513551111133489

Macleod, G., Mcfarlane, B., \& Davis, C. H. (1997). regions in Canada and Mexico.

Marshall, Alfred (1987). Princípios de economia: volume 1. São Paulo: Nova Cultural.

Marx, Karl (1985). O capital. São Paulo: Nova Cultural

Matlay, H. (2011). The influence of stakeholders on developing enterprising graduates in UK HEIs. International Journal of Entrepreneurial Behavior \& Research, 17(2), 166-182. https://doi.org/10.1108/13552551111114923

Mills, C. E. (2012). Navigating the interface between design education and fashion business startup. Education + Training, 54(8), 761-777. https://doi.org/10.1108/00400911211274873

Minard, C. S. L. (2009). Valuing entrepreneurship in the informal economy in Senegal. Social 


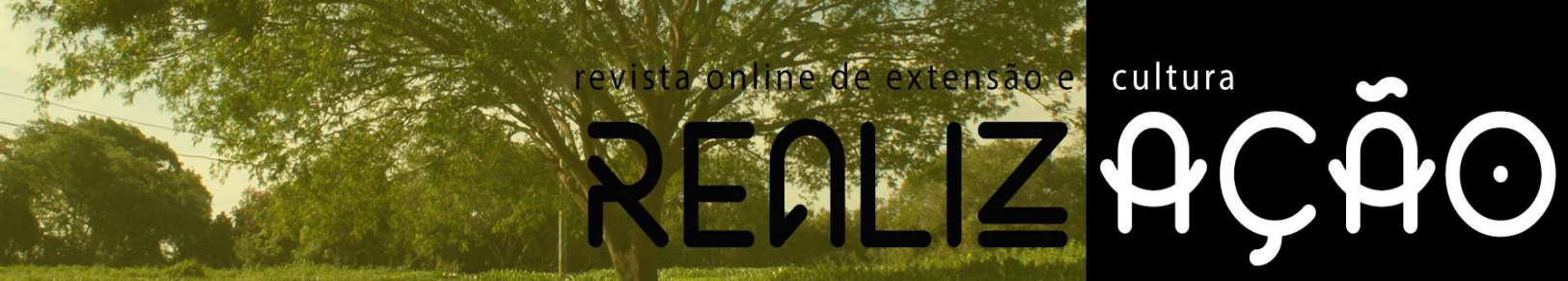

Enterprise Journal, 5(3), 186-209. https://doi.org/10.1108/17508610911004304

Modderman, E., Gorter, C., Dalhuisen, J., \& Nijkamp, P. (2007). Labour manoeuvrability and economic performance: A test on township village enterprises in China. International Journal of Social Economics, 34(4), 220-236. https://doi.org/10.1108/03068290710734190

Newbery, R., \& Bosworth, G. (2010). Home-based business sectors in the rural economy. Society and Business Review, 5(2), 183-197. https://doi.org/http://dx.doi.org/10.1108/17465681011055596

Niu, D., Jiang, D., \& Li, F. (2010). Higher education for sustainable development in China. International Journal of Sustainability in Higher Education, 11(2), 153-162. https://doi.org/10.1108/14676371011031874

Peltier, J. W., \& Scovotti, C. (2010). Enhancing entrepreneurial marketing education: the student perspective. Journal of Small Business and Enterprise Development, 17(4), 514-536. https://doi.org/10.1108/14626001011088705

Piperopoulos, P. (2012). Could higher education programmes, culture and structure stifle the entrepreneurial intentions of students? Journal of Small Business and Enterprise Development, 19(3), 461-483. https://doi.org/10.1108/14626001211250162

Quinn, B., \& Wennes, G. (2008). Mind-sets, mirrors and mid-career education. International Journal of Public Sector Management, $21(4), \quad 353-367$. https://doi.org/10.1108/09513550810880232

Rabayah, K. S., \& Sartawi, B. (2008). Enhancing the labour market prospects of ICT students in a developing country. Education and Training, 50(3), 244-259. https://doi.org/10.1108/00400910810874017

Roper, J., \& Cheney, G. (2005). The meanings of social entrepreneurship today. Corporate Governance: The International Journal of Business in Society, 5(3), 95-104. https://doi.org/10.1108/14720700510604733

Sagie, A., \& Weisberg, J. (2001). The transformation in human resource management in Israel. International Journal of Manpower, 22(3), 226-234. https://doi.org/10.1108/01437720110398275

Samwel Mwasalwiba, E. (2010). Entrepreneurship education: a review of its objectives, teaching methods, and impact indicators. Education + Training (Vol. 52). https://doi.org/10.1108/00400911011017663

Schütz, Rosalvo. Economia Popular Solidária: novos desafios para a educação popular. In: Vários Autores; Série Le Monde Diplomatique Brasil. (Org.). Desafio da economia solidária. São Paulo: Editora e Livraria Instituto Paulo Freire, 2008, v. 04, p. 19-52.

Shenkin, M., \& Coulson, A. B. (2007). Accountability through activism: learning from Bourdieu. Accounting, Auditing \& Accountability Journal, 20(2), 297-317. https://doi.org/10.1108/09513570710741037 
Shurville, S., Browne, T., Whitaker, M., Shurville, S., \& Browne, T. (2009). Accommodating the newfound strategic importance of educational technologists within higher education: A critical literature review. Campus-Wide Information Systems, 26(3), 201-231. https://doi.org/10.1108/10650740910967384

Stanworth, J., \& Stanworth, C. (1990). Small Firms Policy and its Regional Implications in Britain. International Journal of Manpower, 11(6), 8-16. https://doi.org/10.1108/01437729010111085

Sullivan, R. S. (2011). Business schools and the innovation conundrum. Journal of Management Development, 30(5), 492-498. https://doi.org/10.1108/02621711111133000

Thomas, H. (2007). An analysis of the environment and competitive dynamics of management education. Journal of Management Development, 26(1), 9-21. https://doi.org/10.1108/02621710710720040

$\mathrm{T}$

hompson, J. L. (1999). The world of the entrepreneur - a new perspective. Journal of Workplace Learning, 11(6), 209-224. https://doi.org/10.1108/13665629910284990

TRANFIELD, D., DENYER, D., \& SMART, P. (2003). Towards a Methodology for Developing Evidence-Informed Management Knowledge by Means of Systematic Review. British Academy of Management, 14, 207-222.

Turker, D., \& Sonmez Selcuk, S. (2009). Which factors affect entrepreneurial intention of university students? Journal of European Industrial Training, 33(2), 142-159. https://doi.org/10.1108/03090590910939049

Williams, C. C. (2011). Entrepreneurship, the informal economy and rural communities. Journal of Enterprising Communities: People and Places in the Global Economy, 5(2), 145-157. https://doi.org/10.1108/17506201111131578

Williams, C. C., \& Windebank, J. (2006). Harnessing the hidden enterprise culture of advanced economies. International Journal of Manpower, 27(6), 535-551. https://doi.org/10.1108/01437720610690473

Wilson, L. a. (2004). What a difference a decade makes: transformation in academic library instruction. Reference Services Review, 32(4), 338-346. https://doi.org/10.1108/00907320410569680

$\mathrm{Xu}$, L. (2009). Business incubation in China. Management Research Review, 33(1), 90-99. https://doi.org/10.1108/01409171011011599

Zhou, C. (2008). Emergence of the entrepreneurial university in evolution of the triple helix The case of Northeastern University in China. Journal of Technology Management in China, 3(1), 109126. https://doi.org/10.1108/17468770810851539 